\title{
Biopsia hepática y ecografía
}

La introducción de la ecografía en la práctica clínica supuso la reconsideración de

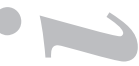
la sistemática diagnóstica en numerosas enfermedades digestivas y, muy especialmente, en las que afectan al hígado y a las vías biliares. De hecho marcó el inicio de la pérdida de protagonismo de la laparoscopia, hasta entonces método de utilización prioritaria en los países de la Europa continental y cambió el procedimiento por el que se guiaba la punción-biopsia del hígado, en contraposición a la biopsia a ciegas cuyo sólo nombre promovía inquietud en pacientes y médicos, a pesar de las escasas complicaciones derivadas de la misma. Inicialmente, incluso, se consideró la posibilidad de que la ecografía sustituyera en la mayoría de los casos al diagnóstico anatomopatológico. Décadas después de la generalización de los estudios sonográficos, y a pesar de la mejora continua de su tecnología, la biopsia sigue siendo imprescindible en numerosas enfermedades del hígado $(1,2)$ y se ha desarrollado una relación de complementariedad entre ambas técnicas, especialmente en dos aspectos:

-Identificación de riesgos antes de realizar biopsia. Se acepta que los pacientes que van a ser sometidos a punción-biopsia hepática deben disponer de un estudio ecográfico reciente o realizado inmediatamente antes de la toma de tejido. Esta metodología evita la punción de lesiones localizadas (quistes, hemangiomas) o de vías biliares intrahepáticas dilatadas, que no pueden ser detectadas mediante percusión, arma fundamental para la realización a ciegas (3).

-Elección de un punto de punción seguro. Este aspecto, confiado exclusivamente al hallazgo de una zona de matidez o submatidez (generalmente en uno de los últimos espacios intercostales derechos) en la modalidad a ciegas (3), ha sufrido un cambio fundamental con el uso de la ecografía, que permite elegir objetivamente el lugar de mayor seguridad, tanto en lo referente a que bajo el mismo se encuentra parénquima hepático como si el espesor de este permite la obtención de una muestra de longitud adecuada, evitando la punción de órganos vecinos. Aparte de la confianza que supone esta guía ecográfica para quien realiza la técnica, los estudios llevados a cabo con la intención de mostrar sus ventajas son de difícil interpretación. En la serie más extensa publicada (4), el uso de guía ecográfica supuso menor tasa de complicaciones y de ingreso hospitalario, lo que justificó el aumento de costes. Aunque era de esperar que el descenso se debiera a menor riesgo de punción de órganos vecinos la diferencia se obtuvo al sumar hemorragia, dolor e hipotensión. No se explica por qué mecanismo pueda impedir la guía sonográfica el desarrollo de hemorragia, que generalmente se produce por punción de pequeños vasos intrahepáticos -no detectables mediante ecografía- o por desgarro de la cápsula (con frecuencia debido a que al paciente no mantiene la apnea durante la punción), sobre todo teniendo en cuenta que el número de intentos -otro factor que determina el riesgo de complicaciones- fue similar en ambos grupos. Tampoco tiene una explicación clara 
la menor incidencia de dolor o hipotensión en los pacientes a los que se realizó biopsia guiada que, por otra parte, no se relacionan con la presencia de complicaciones reales $(3,5,6)$. En una serie publicada posteriormente (7) se confirma la menor incidencia de dolor e hipotensión en los pacientes a los que se realizó biopsia guiada por ecografía, aunque en este caso las complicaciones sí tuvieron relación con el número de intentos realizados para conseguir una muestra de tejido adecuada.

A pesar de las ventajas que supone -según los datos anteriormente citados-para pacientes y médicos la guía sonográfica para la obtención de biopsias hepáticas percutáneas, sorprendentemente el método ha sido aceptado con lentitud. En encuestas realizadas en a la mayoría de los centros de primer nivel en Francia, Suiza, Inglaterra y Gales en un periodo que va desde 1991 a 1997, sólo entre el 34 y el 56\% de las biopsias hepáticas percutáneas fueron obtenidas bajo control ecográfico (8-11).

Un aspecto diferente es el empleo sistemático de la ecografía para identificar las complicaciones derivadas de la punción-biopsia hepática. En este número de la $R e-$ vista Española de Enfermedades Digestivas, Carrera Alonso y cols. (12) informan sobre los datos obtenidos de un análisis prospectivo de 396 punciones (98 con aguja fina) realizando revisión ecográfica 24 horas después. Los resultados confirman la baja tasa de complicaciones incluso si se consideran como tales las que no llegan a tener relevancia clínica, así como la falta de correlación entre signos ecográficos de sangrado (hematomas intrahepáticos o hemoperitoneo) y la aparición de dolor o hipotensión transitorios, hasta el punto que un solo paciente con hematoma cursó con dolor y el tamaño de la colección hemática no era el mayor de los encontrados en la serie. Estos aspectos han sido descritos previamente con metodología y casuística variable.

La aparición de hematomas intrahepáticos después de la biopsia hepática era conocida por los laparoscopistas cuando y donde esta técnica era el vehículo habitual para la punción, ya que su formación podía identificarse, como colecciones subcapsulares, durante el corto periodo de tiempo necesario para vigilar el cese de la hemorragia postpunción que, en cuantía variable, es constante. Ambas incidencias suelen ser asintomáticas y no conllevan habitualmente complicaciones asociadas. Sugano y cols. (13), mediante ecografía sistemática después de la punción, identificaron hematomas en el $20 \%$ de los pacientes biopsiados, sin diferencias según se hubiera realizado a ciegas o dirigida por laparoscopia; sólo 2 de los 24 que los desarrollaron tuvieron dolor, uno de los cuales presentó descenso de la tasa de hemoglobina y condiciones suficientes para aceptar la aparición de una complicación postbiopsia. Menor incidencia (aparecieron hematomas en el 2,3\% de los pacientes biopsiados) encontraron Forssell y cols. (14) sin que en ningún caso se acompañaran de signos clínicos, concluyendo que no es necesaria la exploración sistemática con técnicas de imagen después de realizar la punción. Minuk y cols. (5) detectaron hematomas en el $23 \%$ de los pacientes realizando ecografía 24 horas después de la punción. Siete días después no aumentó la incidencia y se habían resuelto todas las colecciones hemáticas detectadas. Estos autores también confirman la falta de relación entre dolor y/o hipotensión y la presencia de hematoma intrahepático o de sangrado.

Por lo tanto, ante la información de que se dispone no parece necesario, en todos los casos, el estudio ecográfico posterior a la obtención de biopsia hepática percutánea. Los pacientes con trastorno de coagulación en el límite de lo aceptable para la obtención de biopsia percutánea tienen mayor riesgo de sangrado a la cavidad o intrahepático $(15,16)$, en ocasiones de aparición tardía $(17,18)$. En algunas circunstancias concretas se han identificado riesgos no directamente relacionados con los tras- 
tornos de la coagulación (17) (fallo hepático agudo, infiltración hepática linfomatosa, fases avanzadas de la cirrosis hepática, infecciones, ciertos medicamentos), y también en hemodializados. En estos casos, más que seguimiento clínico y ecográfico después de la punción es aconsejable recurrir a la vía transyugular, diseñada originalmente para los pacientes con riesgo de hemorragia. Ante la aparición de signos clínicos (dolor, hipotensión persistentes o descenso de la tasa de hemoglobina) la ecografía permite descartar el desarrollo de complicaciones y, si se han producido, identificar su naturaleza.

Estos aspectos son analizados por Carrera Alonso y cols. (12) llegando a dos conclusiones fundamentales: que no es necesaria la ecografía sistemática después de la práctica de biopsia hepática y que hace falta definir el concepto de complicación, aportando una propuesta en este sentido. Lo último tiene la importancia de identificar las consecuencias habituales de la biopsia, sin repercusión clínica y sin entidad para ser elevadas a la categoría de complicación, y evitar actuaciones que aumentan el coste sanitario.

J. Aguilar Reina

Sección de Hepatología. Servicio de Aparato Digestivo. Hospital Universitario Virgen del Rocío. Sevilla

\section{BIBLIOGRAFÍA}

1. Aguilar Reina J. Indicaciones actuales de la biopsia hepática. Rev And Pat Digest 2005; 28 (ext.) 48-50.

2. Solís Herruzo JA Current indications of liver biopsy. Rev Esp Enferm Dig 2006; 98: 122-9.

3. Aguilar Reina J. Consideraciones actuales acerca de la biopsia hepática. Rev And Pat Digest 2003; 26: 211-4

4. Lindor KD, Bru C, Jorgensen RA, et al. The role of ultrasonography and automatic-needle biopsy in outpatient percutaneous liver biopsy. Hepatology 1996; 23: 1079-83.

5. Minuk GY, Sutherland LR, Wiseman DA MacDonald FR, Ding DL. Prospective study of the incidence of ultrasound-detected intrahepatic and subcapsular hematomas in patients randomized to 6 or 24 hours of bed rest after percutaneous liver biopsy. Gastroenterology 1987; 92: 290-3.

6. Hederstrom E, Forsberg L, Floren CH, Prytz H. Liver biopsy complications monitored by ultrasoud. J Hepatol 1989; 8: 94-8.

7. Farrell RJ, Smiddy PF, Pilkington RM, et al. Guided versus blind liver biopsy for chronic hepatitis C: clinical benefits and costs. J Hepatol 1999; 30: 580-7.

8. Gilmore IT, Burroughs A, Murray-Lyon IM Williams R, Jenkins D, Hopkings A. Indications, methods, and outcomes of percutaneous liver biopsy in England and Wales: and audit by the British Society of Gastroenterology and the Royal College of Physicians of London. Gut 1995; 36: 437-41.

9. Cadranel JF, Rufat P, Degos F. Practices of liver biopsy in France: results of a prospective nationwide survey. For the Group of Epidemiology of the French Association for the Study of the Liver. Hepatology 2000; 32: 477-81.

10. Cadranel JF, Rufat P, Degos F. Practiques de la ponction-biopsie hepatique transparietal en France. Gastroenterol Clin Biol 2001; 25: 77-80.

11. Froelich F, Lamy O, Fried M, Gonvers JJ. Practice and complication of liver biopsy. Results of a nationwide survey in Switzerland. Dig Dis Sci 1993; 38: 1480-4.

12. Carrera Alonso E, García González M, Valer López-Fando P, Plaza Palacios G, López San Román A, Gil Grande L, et al. Estudio prospectivo sobre la utilidad de la ecografía de control tras la realización de pruebas invasivas hepáticas: Biopsia hepática y punción aspiración con aguja fina (PAAF). Rev Esp Enferm Dig 2007; 99 (3): 128-31.

13. Sugano S, Sumino Y, Hatori T, et al. Incidence of ultrasound-detected intrahepatic hematomas due to Trucut needle liver biopsy. Dig Dis Sci 1991; 36: 1229-33.

14. Forssell PL, Bonkowsky HL, Anderson PB, Howell DA. Intrahepatic hematoma after aspiration liver biopsy. A prospective randomized trial using two different needle. Dig Dis Sci 1981; 26: 631-5.

15. Boberg KM, Brosstard F, Egeland T, Egge T, Schrumpf E. Is a prologed bleeding time associated with an increased risk of hemorrage after liver biopsy? Thromb Haemost 1999; 81: 378-81.

16. Terjung B, Lemnitzer I, Dumoulin FL, et al. Bleeding complications after percutaneous liver biopsy. An analysis of risk factors. Digestion 2003; 67: 138-45.

17. Scott DA, Netchvolodoff CV, Bacon BR. Delayed subcapsular hematoma after percutaneous liver biopsy as a manifestation of warfarin toxicity. Am J Gastroenterol 1991; 86: 503-5.

18. Kowdley KV, Aggarwal AM, Sachs PB. Delayed hemorrhage after percutaneous liver biopsy. Role of therapeutic angiography. J Clin Gastroenterol 1994; 19: 50-3. 